\title{
Recovering Streptococcus mutans over restorations by the tray technique - a randomized clinical study
}

\author{
Patricio Vildósola Grez¹, Patricia Palma Fluxa², Paula Zuñiga Saavedra', Eduardo Fernandez Godoy', \\ Osmir Batista de Oliveira Junior ${ }^{3}$, Gustavo Moncada Cortés ${ }^{1}$
}

\author{
'Department of Restorative Dentistry, Operative Dentistry, Dental School, University of Chile, Santiago, Chile \\ 2Department of Medicine and Oral Pathology, Microbiology Dental School, University of Chile, Santiago, Chile \\ ${ }^{3}$ Department of Restorative Dentistry, Araraquara Dental School, UNESP - Univ Estadual Paulista, Araraquara, SP, Brazil
}

\begin{abstract}
Aim: To correlate the tray technique for isolation and counting of Streptococcus mutans over dental restoration with the toothpick technique. Methods: Forty 18-50-year-old patients of both genders with high cariogenic risk were selected. Inclusion criteria were: occlusal restoration evaluated as alpha, according to the Ryge's criteria (examiner' Cohen-Kappa 0.8) and 2 years old maximum age. Each patient provided a saliva sample (control group $S$ ) and two samples of dental biofilm from amalgam or resin-based composite restorations in the same patient, one obtained with the toothpick technique (group TT), and the other from homologous tooth restoration with the tray technique (group TR). This method involves only a direct impression on the occlusal surfaces by a section of a fluoride tray with trypticase yeast extract cysteine sucrose with bacitracin (TYCSB) agar previously prepared and solidified. The samples were incubated at $37^{\circ} \mathrm{C}$ for $48 \mathrm{~h}$ and the colony-forming units (CFU) were counted. The results were statistically analyzed with SPSS software using the linear regression method at $95 \%$ confidence level. Results: TR was able to isolate $S$. mutans, and there was a significant positive correlation $(\mathrm{r}=0.95)$ with TT. There was no significant correlation between TR and control group $\mathrm{S}(\mathrm{r}=0.47)$. Conclusions: TR was able to isolate and count $S$. mutans from the dental biofilm of dental restorations with a significant correlation with the TT group.
\end{abstract}

Keywords: Streptococcus mutans, dental caries, dental restoration.

Received for publication: August 26, 2013

Accepted: November 28, 2013

Correspondence to: Patricio Vildósola Grez

Sergio Livingstone 943, office 304 , Independencia, Santiago, Chile Phone: +56 0229781742 / fax: +56 0229781742 E-mail: patovildo@gmail.com

\section{Introduction}

Caries is one of the most common diseases in the whole world and although the industrialized countries have lowered their rates, it cannot be completely eradicated; therefore a treatment is required, consuming large human and economic resources $^{1,2}$. Among the factors in the complex process of caries disease are the bacteria, which are immersed in cariogenic biofilm in balance with the oral environment ${ }^{3-5}$. This balance can be altered by microbial carbohydrate intake, increasing the population of acidogenic bacteria responsible for demineralization and destruction of hard tooth tissue, thereby causing the caries lesion ${ }^{6}$. Such ecological disruptions of the microbial community are prone to occur because of microbial acid-induced adaptation and selection processes 5 . 
In this cariogenic biofilm are found mutans streptococci, that are the main etiologic agents of dental caries in humans $s^{7,8}$ and are classified into seven species. Out of these, Streptococcus mutans (S. mutans), is the most frequently isolated pathogen from biofilm ${ }^{9}$. The levels of $S$. mutans in dental plaque samples have been shown to be associated with the development of caries and their detection has been employed to predict caries risk, to monitor caries activity ${ }^{10}$ and also play an important role in the secondary caries ${ }^{11}$, because dental caries is a localized disease of the teeth ${ }^{12}$.

In order to facilitate the isolation of this microorganism selective Gold culture media have been used ${ }^{13}$, and different methods have been proposed in an attempt to simplify $S$. mutans culture in laboratory for its detection and quantification ${ }^{14}$. The $S$. mutans samples are processed and inoculated in selective culture media, such as mitis salivarius agar with bacitracin (MSB) and trypticase yeast extract cysteine sucrose with bacitracin (TYCSB) ${ }^{15}$.

Several sampling methods for $S$. mutans have been proposed, such as counting $S$. mutans in saliva or taking direct samples of biofilm from teeth or restorations. In the case of counting $S$. mutans from saliva, it can be done stimulating or not the saliva ${ }^{16}$. This method is easy and inexpensive, but it presents some limitations, e. g. a person with low counts of $S$. mutans in saliva could have high concentrations of $S$. mutans on a restoration ${ }^{17}$. Therefore, it has been suggested that the direct identification of the $S$. mutans colonies on the restoration margins allow a better assessment of the presence of the bacteria and a more accurate estimation of the failure risk of dental restorations ${ }^{16}$. For this reason, a few years ago, Wallman and Krasse ${ }^{18}$ proposed a more precise method for counting $S$. mutans on restorations. They suggested that plaque samples should be collected from restorations using the tip of a sterile wooden instrument (a toothpick). It is a simple method of collection to perform in the dental office. However, the authors pointed to a great disadvantage: this technique often results in underestimating the presence of microorganisms, which may not be accessed by the toothpick, due to its shape and size.

The toothpick technique was one of the most used methods. The present paper describes a new method that allows the identification and quantification of $S$. mutans directly from restored teeth with a tray technique by a previously solidified selective medium.

The objective of this study was to evaluate the recovery of the tray technique (TR) for the isolation and counting of $S$. mutans populations on occlusal surfaces of restored teeth and correlate it with the toothpick method. The hypothesis tested is that the TR is able to the recover $S$. mutans from occlusal surfaces of restorations and has correlation with the toothpick method.

\section{Material and methods}

\section{Experimental design}

This experimental, randomized and blind study was part of project PRI-ODO 11-02 and was approved by the Ethics
Committee of the Dental School at Universidad de Chile protocol 2011/07. The objectives of this study were explained to all participants, who provided written consent. The study was conducted in full accordance with ethical principles, including the World Medical Association's Declaration of Helsinki (2002) and local regulations.

A sample size equal to or greater than 40 restored teeth was determined with a confidence interval of $95 \%(a=0.05)$ and a statistical power of 0.9 (G*Power 3.1 Software) $)^{19}$.

A total of 584 patients of the Operative Dentistry Clinic of the Dental School, Universidad de Chile were examined and 40 patients were randomly selected to participate using the NCSS PASS 2008, v08.0.15 software, and their data were stored in a Microsoft Office Excel (Version 2007) spreadsheet.

\section{Inclusion Criteria}

- Age between 18 and 50 years

- High caries risk patients according to the Cariogram software

- Occlusal restoration of the same material in the contralateral arch

- Full dentition ( $>28$ teeth)

- Amalgam and resin-based composite restorations

- Restorations must have Alpha value in five parameters according to the United States Public Health Service (USPHS/ RYGE) criteria ${ }^{20}$ (Table 1), assessed by a calibrated clinician $($ Cohen Kappa $=0.8)$

- Dental restorations placed within a maximum period of 2 years in the Operative Dentistry Clinic of the Dental School, Universidad de Chile

- Occlusal restorations in premolars and molars of no more than $1 / 3$ of the intercuspal distance

\section{Exclusion Criteria}

- Bravo and Charlie restorations according to the USPHS/ RYGE criteria (Table 1)

- Patients taking drugs that are proven to reduce salivary flow, such as antidepressants, narcotics, antihistamines, antihypertensive, antiemetic and diuretics

- Patients undergoing treatment with mouth rinses and/or other oral antiseptics and/or toothpaste with fluoride concentrations greater than or equal to $2500 \mathrm{ppm}$ fluoride ions at the time of the study or in the previous last 3 months - Patients taking antibiotics at the time of the study or in the last 3 months

- Patients taking immunosuppressive drugs (corticosteroids) - Patients classified as ASA III according to the American Society of Anesthesiologists,

- Patients who chew gum at least four days per week ${ }^{21}$

- Patients with a physical disability that precludes them being responsible for their own hygiene

\section{Sampling and isolation of S. mutans}

Selected patients were instructed on Bass technique, and their performance was evaluated one week later, prior to sample taking, using the plaque index (modified by Loe).

All samples were collected in the morning between 10:00- 
Table 1. Ryge Criteria

\begin{tabular}{llll}
\hline $\begin{array}{l}\text { Clinical characteristic } \\
\text { Marginal Adaptation }\end{array}$ & $\begin{array}{l}\text { Alpha } \\
\text { Explorer does not catch or has one way catch } \\
\text { when drawn across the restoration/tooth interface }\end{array}$ & $\begin{array}{l}\text { Bravo } \\
\text { Explorer falls into crevice when drawn } \\
\text { across the restoration/tooth interface }\end{array}$ & $\begin{array}{l}\text { Charlie } \\
\text { Dentin or base is exposed along the } \\
\text { margin }\end{array}$ \\
\hline Anatomic Form & $\begin{array}{l}\text { The general contour of the restorations follows } \\
\text { the contour of the tooth }\end{array}$ & $\begin{array}{l}\text { The general contour of the restoration } \\
\text { does not follow the contour of the tooth }\end{array}$ & The restoration has an overhang \\
\hline Surface Roughness & $\begin{array}{l}\text { The surface of the restoration does not have any } \\
\text { surface defects }\end{array}$ & $\begin{array}{l}\text { The surface of the restoration has minimal } \\
\text { surface defects }\end{array}$ & $\begin{array}{l}\text { The surface of the restoration has severe } \\
\text { surface defects }\end{array}$ \\
\hline Secondary Caries & There is no clinical diagnosis of caries & N/A & $\begin{array}{l}\text { Clinical diagnosis of caries at restoration } \\
\text { margin }\end{array}$ \\
\hline Luster of Restoration & $\begin{array}{l}\text { The restoration surface is shiny and has an } \\
\text { enamel-like, translucent surface }\end{array}$ & $\begin{array}{l}\text { The restoration surface is dull and } \\
\text { somewhat opaque }\end{array}$ & $\begin{array}{l}\text { The restoration surface is distinctly dull } \\
\text { and opaque and is esthetically unpleasing }\end{array}$ \\
\hline
\end{tabular}

13:00 am. On one side of the mouth, a sample of plaque was taken from the restoration using the tray technique (TR), while on the opposite side the sample was obtained using the toothpick technique (TT). While biofilm samples from restorations were obtained by the TR and TT methods from each patient, non-stimulated whole saliva samples were collected into sterile glass beaker for $5 \mathrm{~min}^{22}$.

In the TR technique, biofilm on the surface of restorations was collected using a disposable fluoride gel application tray (Deepak Products Inc., Miami, FL, USA). Each tray was sterilized in a type II biosafety hood (Esco Technologies, Inc., Harboro, PA, USA) under ultraviolet light for $20 \mathrm{~min}$ and filled with TYCSB agar. The composition of the media per litre was:

Casein (15.0 g), yeast extract (5.0 g), L-cysteine (200 $\mathrm{mL}$ ) (Difco Laboratories Inc., Detroit, MI, USA), sodium sulfite $(0.1 \mathrm{~g})$, sodium chloride $(1.0 \mathrm{~g})$, sodium phosphate 12 hydrate $(2.0 \mathrm{~g})$, sodium bicarbonate $(2.0 \mathrm{~g})$, sodium acetate trihydrate (20.0 g), sucrose (50.0 g), agar (15.0 g), distilled water (qsp), $0.2 \mathrm{U} / \mathrm{mL}$ bacitracine ${ }^{23}$. The biofilm collected with TT was removed from the surfaces with the tip of a sterile, triangular, wooden toothpick. Patient was asked to rinse the mouth with water, and the teeth were isolated using cotton rolls and gently air dried for $5 \mathrm{~s}$ to avoid contamination with saliva. The TT samples were transferred to $500 \mu \mathrm{L}$ of RTF (reduced transport fluid) and stored at $4{ }^{\circ} \mathrm{C}$ until laboratory processing.

All samples were transported to the laboratory and manipulated on the same day. Saliva was homogenized in a Vortex homogenizer (Maxi Mix II type 37600, Mixer Barnstead Thermolyne, Dubuque, IA, USA). In addition to the trays used for sample collection, trays loaded with TYCSB were incubated at $37{ }^{\circ} \mathrm{C}$ (ZDP-A2080; LabTech Co., Namyangju-city, Korea) for $24 \mathrm{~h}$ as a quality control.

All of these processes were performed at Oral Microbiology Laboratory, Department Medicine of Oral Pathology, Dental School, Universidad de Chile.

\section{Bacteriological Procedures}

The plaque samples obtained by the toothpick technique were homogenized in a Vortex mixer (Maxi Mix II type 37600
Mixer, Barnstead Thermolyne) for $45 \mathrm{~s}$. Then, $100 \mu \mathrm{L}$ of the homogenate of plaque samples was added to a tube with 900 $\mu \mathrm{L}$ of phosphate ( $\mathrm{pH}$ 7.2) and shaken for $15 \mathrm{~s}$ in a Vortex. A $100 \mu \mathrm{L}$ aliquot was obtained after the first dilution and added to a second tube with $900 \mu \mathrm{L}$ of phosphate buffer. Thus, dental plaque dilutions of $1: 10$ and $1: 100$ were obtained.

The same procedure was performed with the saliva samples to obtain dilutions of 1:1000 and 1:10 000.100 $\mu \mathrm{L}$ of each of the diluted plaque and saliva samples was plated in TYCSB agar plates using a micropipette. The entire plating process was performed under sterile conditions in a hood or type II biosafety cabinet. The plates were placed in a candle jar system and incubated at $37{ }^{\circ} \mathrm{C}$ for $48 \mathrm{~h}$.

\section{Isolation and identification of $S$. mutans}

From the different samples (TR, TT and saliva), S. mutans colonies were identified based on colony morphology (macroscopic) and adherence to the agar colonies observed under a stereomicroscope (Stemi 2000; Carl Zeiss Microscopy, Thornwood, NY, USA) with a light source (Schott KL 1500, Carl Zeiss Microscopy).

The $S$. mutans count was expressed as colony forming units (CFU) of $S$. mutans from the plates and trays with TYCSB agar. Then, the selected colonies that were compatible with $S$. mutans adhesion and morphology characteristics were suspended in Todd-Hetwitt broth (Difco Laboratories Inc.) and incubated at $37{ }^{\circ} \mathrm{C}$ for $48 \mathrm{~h}$. The colonies were then subjected to biochemical tests to identify the species of Mutans streptococci and distinguish $S$. mutans from Streptococcus sobrinus.

Biochemical tests were performed, including raffinose fermentation, melibiose and esculin hydrolysis. When all three are positive they indicate presence of $S$. mutans ${ }^{24}$.

After $48 \mathrm{~h}$, each incubated broth sample was centrifuged (BD Sero-Fuge 2001, Clay-Adams Becton, Dickinson and Co., Sparks, MD, USA) for $5 \mathrm{~min}$ at approximately $1500 \mathrm{rpm}$ to obtain a pellet. The pellet was resuspended in $450 \mu \mathrm{L}$ of phosphate buffer ( $\mathrm{pH}$ 7.2) to obtain a near McFarland 5: 100 $\mu \mathrm{L}$ of this suspension was inoculated in esculin (Brain Heart infusion, $1 \%$ esculin. Difco Laboratories Inc.) in raffinose 
(thioglycolate without dextrose and no indicator, $1 \%$ of raffinose. Difco Laboratories) and melibiose (thioglycolate without dextrose and no indicator, $1 \%$ of melibiose. Difco, Laboratories) and incubated for $24 \mathrm{~h}$ at $37{ }^{\circ} \mathrm{C}$. Subsequently, two drops of ferric ammonium citrate were added to melibiose and raffinose broths, respectively.

The hydrolysis of esculin is considered positive if the broth quickly exhibits black color, and the fermentation of raffinose and melibiose was identified by yellow color, which indicated a positive test. Both situations confirm the diagnosis of $S$. mutans.

Counting of $S$. mutans $\mathrm{CFU}$ from the samples was performed by a single calibrated examiner (Cohen-Kappa $>0.8$ ).

\section{Statistical analysis}

A single blind statistician analyzed the results using the Statistical Package for Social Sciences (SPSS) software for Windows, version 15.0 (SPSS Inc., Chicago, IL, USA).

Analyses were used to verify the normality of the data distribution and the homogeneity of variance with simple linear regression correlation. The significance level was set at $5 \%$

\section{Results}

\section{Identification and isolation of $S$. mutans}

Based on the bacterial culture and isolation from dental plaque samples by the TR, TT and S methods in TYCSB agar, isolated colonies were obtained with macroscopic properties and adhesion characteristics that were indicative of S. mutans. (Figs. 1 and 2)

The smears of the colonies showed Gram staining of selected cocci forms: they were Gram positive and arranged in chains, which is typical of $S$. mutans bacteria.

Tests for esculin hydrolysis and fermentation of raffinose and melibiose were positive for all isolates; therefore, the presence of $S$. sobrinus was not detected.

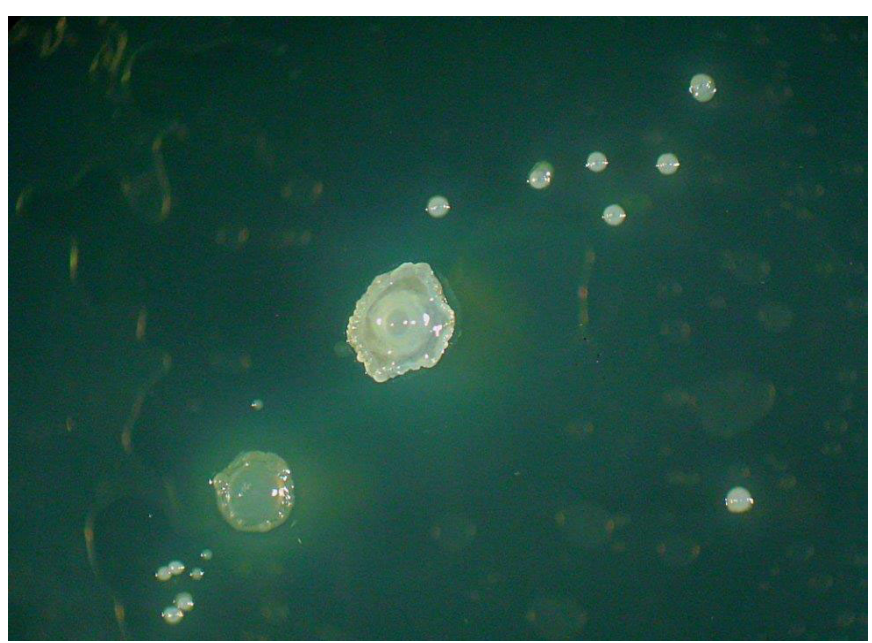

Fig. 1. Colonies of S.mutans on TYCSB agar exhibited a whitish, rough surface and adhered to agar (observed under $4 \mathrm{x}$ magnification)
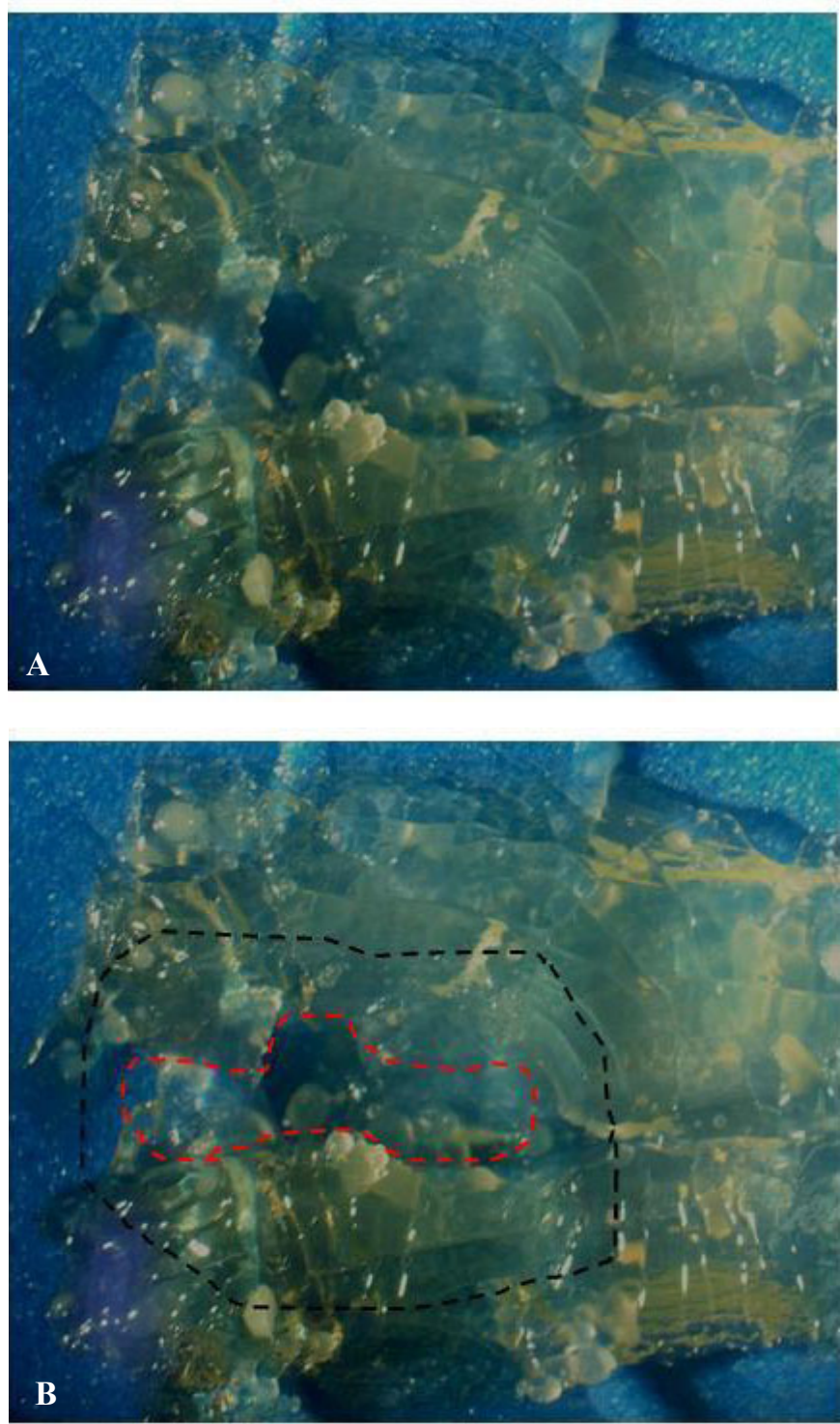

Fig. 2. Bacterial isolation from dental plaque samples using TR (A) Colonies of $S$, mutans had a round, smooth, crystalline appearance and adhered to the imprint of the occlusal surface of the restored tooth. (B) The red line represents the outline of the occlusal surface of the tooth.

\section{Quantification of S. mutans}

Approximately $87.5 \%$ (35 out of 40 ) of the TR, TT and $\mathrm{S}$ samples showed the presence of $S$. mutans. CFU average was TR $7.98( \pm 8.70) \mathrm{CFU} / \mathrm{cm}^{2}$, TT $3.13( \pm 4.77) \times 10^{3} \mathrm{CFU} /$ $\mathrm{mL}, \mathrm{S} 5.05( \pm 6.40) \times 10^{5} \mathrm{CFU} / \mathrm{mL}$.

\section{TR and TT Correlation}

A simple linear regression was calculated to determine the correlation between the $S$. mutans counts obtained by TR and TT. The obtained result was $\mathrm{r}=0.95$ (Fig. 3). A positive value of $r$ greater than or equal to 0.65 was considered a significant correlation.

The correlation between the counts of $S$. mutans from the plaque samples of the restorations obtained by TR and $\mathrm{S}$ was positive but not significant $(\mathrm{r}=0.47)$ (Fig. 4). 


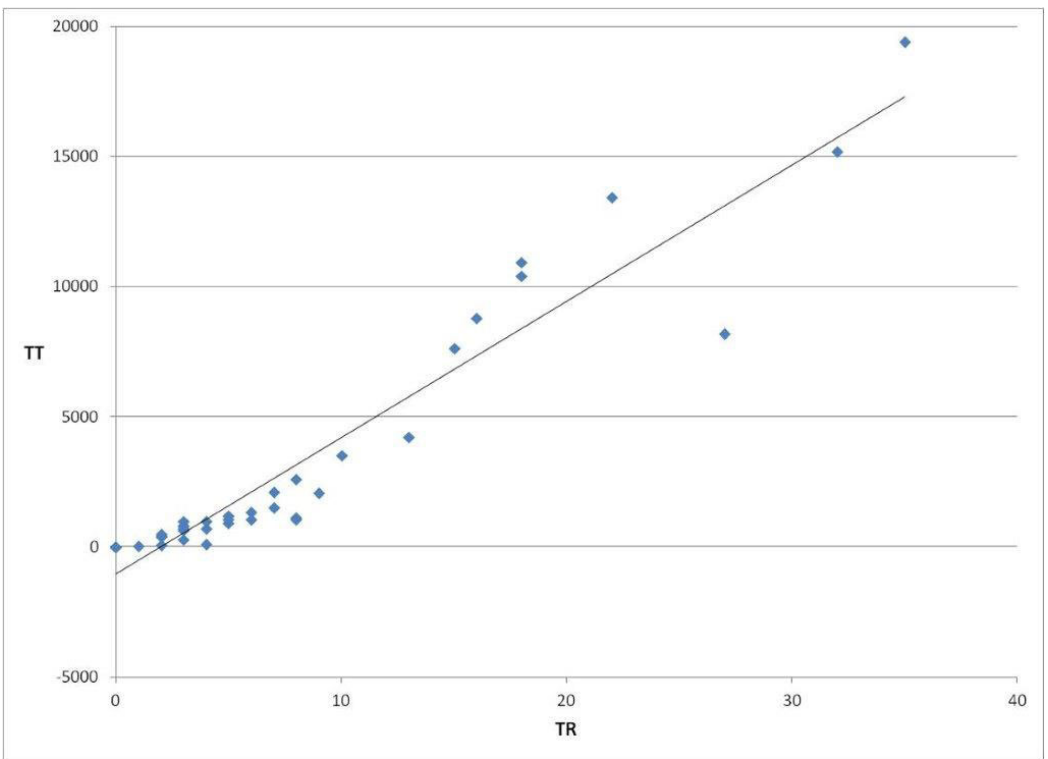

Fig. 3. Correlation between the counts of S. mutans by TT and TR from dental plaque samples from restorations.

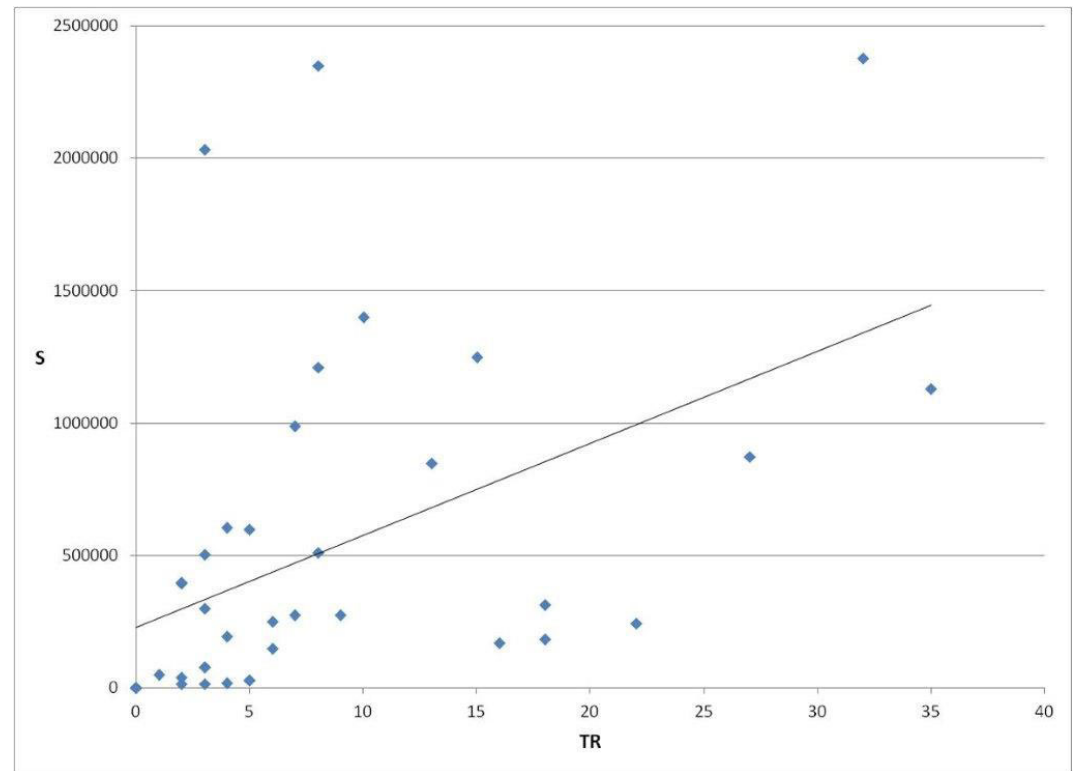

Fig. 4. Correlation between the counts of $S$. mutans by $S$ and TR from dental plaque samples on restorations.

\section{Discussion}

This study demonstrated a significant correlation between TR and TT methods of collecting plaque samples of $S$. mutans in occlusal restorations in a selective medium for S. mutans (TYBSC). Additionally, a positive but nonsignificant correlation was observed between the results of TR and saliva, and similar results were observed when correlating the results of TT and saliva. This result may be explained mainly by the difficulty of removing the biofilm from different areas of the teeth by chewing on paraffin wax ${ }^{17}$.

In this research the patients were standardized by sampling on restorations with Alpha rating, mainly to homogenize the samples. However it would be interesting to investigate what happens with patients who have Bravo rating restorations, because they have a greater biofilm accumulation, therefore a higher caries risk.

It is known that $S$. mutans levels can vary among restorations, even in the same subject ${ }^{11}$. For this reason, Wallman and Krasse proposed the toothpick method that collects $S$. mutans from the restoration's margins ${ }^{18}$. This method has been used extensively and it is considered to be the simplest available method. It is a non-invasive test for monitoring $S$. mutans in dental restorations. Nevertheless, it has been reported that TT could not collect the complete range of the oral microbiota on restorations ${ }^{25}$. The TR is able to recover the bacteria deposited in all the surface occlusal restorations. In addition, TR is the most suitable 
technique for the monitoring of $S$. mutans in dental restorations due to the several advantages of this method relative to TT. For example TR involves a simpler method of microbiological processing due to the elimination of dilution and seeding steps, so it requires fewer logistical resources.

The TR technique involves taking a direct impression of the restoration, and it was very well tolerated by the patients. The possibility of gag reflex was avoided because there was little contact with culture medium and no patient complained of bad taste. Furthermore, it is a non-invasive technique for detecting microorganisms on surfaces, which could be used for monitoring early caries lesions ${ }^{9,26}$ and assessing local risk factors for the development of caries lesions.

In the present study, only TYCSB agar was used due to its high specificity for $S$. mutans, but it is recommended to use other culture media, such as SB or SB-20M to determine more specifically strains of $S$. sobrinus ${ }^{27}$ with the $S$. mutans, because they are an important factor in the development of dental caries s, $^{58,29}$.

This technique may be used for clinical research with new antibacterial materials in a very simple way. Current studies on antibacterial materials are focused on $S$. mutans, but it is necessary to investigate other bacteria that are involved in the caries process. The technique is limited to free dental surfaces and is not indicated for proximal surfaces due to the low resistance of the culture medium to tearing and its deformation, which impedes the access to the other area of interest.

The following conclusions can be drawn based on the obtained results: 1 . The tray technique may recover and count $S$. mutans on occlusal surfaces of dental restorations, having a positive correlation with TT; 2 . The tray technique may be considered as less time consuming and simpler than the toothpick technique for being a direct and non-invasive method of detecting S. mutans.

\section{Acknowledgements}

The authors are indebted to Dra. Marta Estela Saravia of Department of Preventive Dentistry, School of Dentistry National Of Tucumán, Argentina for her helpful assistance in the microbiological procedures.

\section{References}

1. Marcenes W, Kassebaum NJ, Bernabé E, Flaxman A, Naghavi M, Lopez A, et al. Global burden of oral conditions in 1990-2010: a systematic analysis J Dent Res. 2013; 92: 592-7.

2. Richards D. Oral diseases affect some 3.9 billion people Evid Based Dent. 2013; 14: 35.

3. Nyvad B, Crielaard W, MiraA, Takahashi N, Beighton D. Dental caries from a molecular microbiological perspective. Caries Res. 2013; 47: 89-102.

4. Takahashi N, Nyvad B. Caries ecology revisited: microbial dynamics and the caries process. Caries Res. 2008; 42: 409-18.

5. Takahashi N. Nyvad B. The role of bacteria in the caries process: ecological perspectives. J Dent Res. 2011; 90: 294-303.

6. Beighton $\mathrm{D}$. The complex oral microflora of high-risk individuals and groups and its role in the caries process. Community Dent Oral Epidemiol. 2005; 33: $248-55$.
7. Aas JA, Griffen AL, Dardis SR, Lee AM, Olsen I, Dewhirst FE, et al. Bacteria of dental caries in primary and permanent teeth in children and young adults J Clin Microbiol. 2008; 46(4): 1407-17.

8. Preza D, Olsen I, Aas JA, Willumsen T, Grinde B, Paster BJ. Bacterial profiles of root caries in elderly patients. J Clin Microbiol. 2008; 46: 2015-21.

9. Tanzer JM, Livingston J, Thompson AM. The microbiology of primary dental caries in humans. J Dent Educ. 2001; 65: 1028-37.

10. Fontana M, Zero DT. Assessing patients' caries risk J Am Dent Assoc. 2006; 137: 1231-9.

11. Bentley CD, Broderius CA, Drake CW, Crawford JJ. Relationship between salivary levels of mutans streptococci and restoration longevity. Caries Res. 1990; 24: 298-300.

12. Fejerskov O, Nyvad B, Kidd EAM. Pathology of dental caries. In: Fejerskov O, Kidd EAM, editors. Dental caries. The disease and Its clinical management. 2nd ed. Oxford: Blackwell Munksgaard; 2008. p. 19-48.

13. Gold OG, Jordan HV, Van Houte J. A selective medium for Streptococcus mutans. Arch Oral Biol. 1973; 18: 1357-64.

14. Westergren $G$, Krasse B. Evaluation of a micromethod for determination of Streptococcus mutans and Lactobacillus infection. J Clin Microbiol. 1978: 82-3.

15. Wan AK, Seow WK, Walsh LJ, Bird PS. Comparison of five selective media for the growth and enumeration of Streptococcus mutans. Aust Dent J. 2002; 47: 21-6.

16. Park JH, Tanabe $Y$, Tinanoff N, Turng BF, Lilli H. Minah GE. Evaluation of microbiological screening systems using dental plaque specimens from young children aged 6-36 months. Caries Res. 2006; 40: 277-80.

17. Wallman $C$, Krasse $B$. Mutans streptococci in margins of fillings and crowns J Dent. 1992; 20: 163-6.

18. Wallman C, Krasse B. A simple method for monitoring mutans streptococci in margins of restorations. J Dent. 1993; 21: 216-9.

19. Faul F, Erdfelder E, Buchner A, Lang AG. Statistical power analyses using $G^{*}$ Power 3.1: tests for correlation and regression analyses. Behav Res Methods. 2009; 41: 1149-60.

20. Ryge G. Clinical criteria. Int Dent J. 1980; 30: 347-58.

21. Zibell S, Madansky E. Impact of gum chewing on stress levels: online self-perception research study. Curr Med Res Opin. 2009; 25: 1491-500.

22. Silva TC, Valarelli TM, Sakal VT, Tessarolli V, Machado MAAM. Oral Antibacterial effect of chlorhexidine treatments and professional prophylaxis in children. Braz J Oral Sci. 2013; 12: 132-7.

23. Van Palenstein HWH, ljsseldijk M, Huis in 't Veld JH. A selective medium for the two major subgroups of the bacterium Streptococcus mutans isolated from human dental plaque and saliva. Arch Oral Biol. 1983; 28: 599-603.

24. Coykendall AL. Classification and identification of the viridans streptococci Clin Microbiol Rev. 1989; 2: 315-28.

25. Wennerholm K, Lindquist B, Emilson CG. The toothpick method in relation to other plaque sampling techniques for evaluating mutans streptococci. Eur J Oral Sci. 1995; 103: 36-41.

26. Coogan MM, Mackeown JM, Galpin JS, Fatti LP. Microbiological impressions of teeth, saliva and dietary fibre can predict caries activity. $J$ Dent. 2008; 36: 892-9.

27. Saravia ME, Nelson-Filho P, Silva RA, De Rossi A, Faria G, Silva LA Emilson CG. Recovery of mutans streptococci on MSB, SB-20 and SB20M agar media. Arch Oral Biol. 2013; 58: 311-6.

28. Saravia ME, Nelson-Filho P, Ito IY, da Silva LA, da Silva RA, Emilson CG. Morphological differentiation between $S$. mutans and $S$. sobrinus on modified SB-20 culture medium. Microbiol Res. 2011;166: 63-7.

29. Peterson SN, Snesrud E, Schork NJ, Bretz WA. Dental caries pathogenicity: a genomic and metagenomic perspective. Int Dent J. 2011; 61(Suppl 1): 11-22. 